\title{
1995 HEWLETT-PACKARD EUROPHYSICS PRIZE
}

\section{Fundamentals with a Profound Impact}

The 1995 Hewlett-Packard Europhysics Prize for outstanding achievements in condensedmatter physics has been awarded to Yakir Aharanov, Tel Aviv University, the University of South Carolina and Boston University, and to Michael V. Berry, Bristol University, for introducing fundamental concepts in physics that have had a profound impact on condensedmatter science. Both are distinguished scientists who have made fundamental contributions in a wide area of theoretical physics. Some of these continue to have a strong impact namely, the Aharanov-Bohm effect first predicted in 1956 and the Berry's phase first introduced in 1984 of which the Aharanov-Bohm effect can be considered to be a special case.

Demonstrating the Aharanov-Bohm effect. The conductivity of long, thin-walled cylinders (radius $0.8 \mu \mathrm{m}$; length $\approx 2 \mu \mathrm{m}$ ) made of a normal metal $(\mathrm{Mg})$ is an oscillatory function of the magnetic flux which passes through the cylinder. [After Sharvin \& Sharvin, 1982]

The Aharanov-Bohm effect is at the root of flux quantization, the Josephson effect and the quantum Hall effect, all of great importance in condensed-matter physics. Aharanov and Bohm predicted that charge particles moving through the vicinity of a shielded magnet such that they experience no force due to the magnet will nonetheless experience a phase change in the wave function which can be observed in an interference experiment. The potentials (in the case of this example the vector potentials) and not the fields act on the particles revealing the physical significance of potentials.

The Aharanov-Bohm effect has been observed in the interference effects involving electrons travelling on opposite sides across a solenoid (Chambers) and more recently between electrons moving through and outside a toroidal magnet coated with a superconductor (Tonomura et al.). One is sure in the latter experiment that the magnetic field strength is zero in the region traversed by both trajectories. The explanation of the interference effects lies in the dependence of the phase of the wave functions on the geometric trajectory followed, above and beyond a dynamical phase difference which

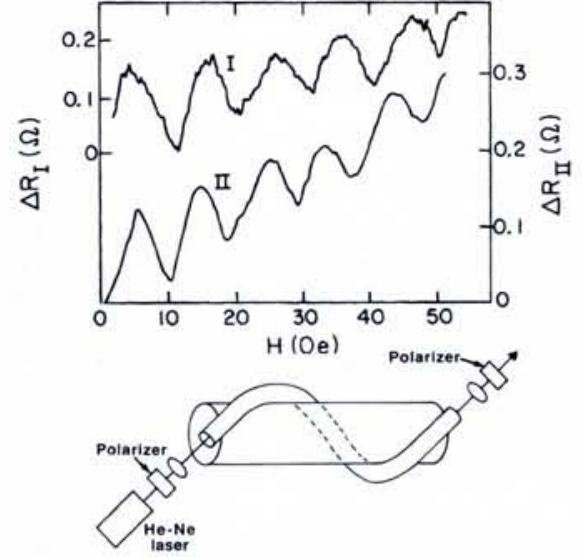

Measuring Berry's phase. The angle of rotation of linearly polarized light from a neonhelium laser on passing through a helical, single-mode optical fibre is a measure of Berry's geometrical phase [After Tomita \& Chiao, 1986]

depends only on the elapsed time. The Aharanov-Bohm effect is not limited to the influence of the magnetic potential vector, but also occurs in the presence of scalar electric potentials. More recently, the effect has also been observed by Sharvin and Sharvin for electrons moving in a long, thinwalled cylinder in the presence of a vector potential by observing the change in the electrical resistance as a function of the vector potential (see figure)

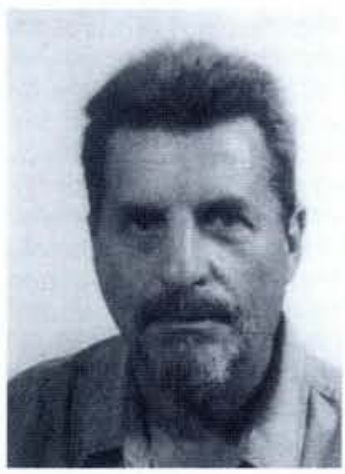

Yakir Aharanov (left) has held a joint appointment as Professor of Physics at Tel Aviv University and at the University of South Carolina since 1973, and is a long-term visiting professor at Boston University. He studied at the Technion, Haifa, and was awarded a Ph.D. by Bristol University in 1960. After fours spent as a research associate at Brandeis University, he returned to Israel to become an Assistant Professor and subsequently Associate Professor at Yeshiva University before taking up a joint position as Professor at Tel Aviv University and Yeshiva University. Professor Aharanov won the 1984 Weizmann Prize in Physics and the 1989 Israel National Prize in Physics and is a member of the US National Academy of Sciences.

Michael V. Berry (right) has been the Royal Society Research Professor at the H.H. Wills Physics Laboratory, Bristol University, UK, since 1988 and recently became Cornell University's A.D. White Visiting Professor-at-Large. He studied at Exeter University and at St. Andrews University where he was awarded a Ph.D. in theoretical physics in 1965. Professor Berry then joined Bristol University as a postdoctoral research fellow, becoming Lecturer in Physics in 1967, Reader in 1975 and Professor in 1979. He was awarded the Institute of Physics 1978 Maxwell Medal and Prize and its 1990 Paul Dirac Medal and Prize, the American Physical Society's 1990 Julius Edgar Lilienfeld Prize, and The Royal Society's 1990 Royal Medal. He is a Fellow of the Royal Society of London and a member of Academia Europaea.
In 1984, Berry showed that in an adiabatically varying system in which some parameters after certain time return to their initial values, the wave function obtains, in addition to a dynamical phase factor, a geometrical phase factor which depends only on the path the system described in parameter space. This elementary observation, although essentially quite basic, is really very profound and modifies fundamentally the quantum adiabatic theorem of the late1920 's. According to this theorem, the wave function will instantaneously adjust to a very slowly varying environment such that if the environment returns to its original condition the wave-function will return to its original value multiplied by the dynamical phase factor, which depends only on the duration of the excursion.

According to Berry, it is as if a force has been acting which is similar to the influence of a gauge field. For the Aharanov-Bohm effect, the adiabatic excursion in real space occurs in the presence of a gauge field which generates the extra phase. Berry's phase involves a more general adiabatic excursion in parameter space which can similarly be traced to the influence of a gauge field.

Aside from the Aharanov-Bohm effect, Berry's phase is found in numerous situations, many of them relevant to condensed-matter science. Well-known instances include:

- the splitting observed in magnetic resonance of a spin system in a slowly varying magnetic field;

- nuclear quadrupole resonance in a slowly rotating sample;

- fractional quantizations of molecular pseudo-rotations;

- the enrichment of lattice and molecular dynamics in the Born-Oppenheimer approximation by a new gauge force when, near an electronic degeneracy like that in dynamic Jahn-Teller distortions, effective attractive contributions to the electron-electron interaction can arise in Jahn-Teller molecules such as $C_{60}^{3-}$ which are possibly important for superconductivity in compounds like $\mathrm{K}_{3} \mathrm{C}_{60}$; - photons propagating down a helically wound optical fibre (see figure);

- fractional statistics including, for example, anyons or semions as proposed by some authors in describing high-temperature superconductors as well as the fractional quantum Hall effect;

- the theory of macroscopic polarization in crystalline dielectrics, and so on.

One of the most profound applications of Berry's phase in condensed-matter science perhaps concerns the effective treatment, based on quasi-particles which obey fractional statistics, of the many-body problem for the low-energy scale properties of solids such as high-temperature superconductors.

As far as practical applications of the Aharanov-Bohm effect or Berry's phase are concerned, various microelectronics industries are actively seeking ways to use quantum-interference effects in devices in order to minimize power dissipation. These devices control resistance and voltage by manipulating the wave characteristics of an electron using a potential.

G.A. Sawatzky, Groningen University 